\title{
A Programmable High Speed Vision System with Superscalar PE and Its Parallel Computing Language
}

\author{
Jie Yang, Cong Shi, Xitian Long, Nanjian Wu \\ Institute of Semiconductors, Chinese Academy of Sciences, Beijing, China \\ Email: yangjie@semi.ac.cn
}

Received 2012

\begin{abstract}
Pixel-parallel PE and SIMD architectures are widely used in high-speed image processing to enhance computing power. With fully exploiting the data level parallelism of low- and middle-level image processing, SIMD architecture is able to finish great amount of computation with much less instruction cycle thus satisfy the high-speed system requirement. The main computation parts in those SIMD image processing hardware is known as PE (processing element) and it is responsible for transferring, storing and processing the image data. This paper describes a high-speed vision system with superscalar PE to enhance system performance and its dedicated parallel computing language specifically developed for this vision system. The vision system can achieve motion detection at more than $2000 \mathrm{fps}$ and face detection at more than $100 \mathrm{fps}$ which overwhelms some general serial CPUs in the same applications.
\end{abstract}

Keywords: High-Speed Vision System; SIMD; Superscalar; PE

\section{Introduction}

Researchers have been interested in high-speed vision system for decades [1]. It can be applied in many fields, such as real-time object-tracking, machine vision, industry controls. Traditional machine vision systems which are composed of image sensor and general-purpose processor have heavy I/O load induced by large amount of image data transfer and lack of computational power for low- and middle-level processing. Our previous design [2] using multi-level parallel processors to fully cover low-, middle-, and high-level image processing and with dedicated programming language this design can finish various high-speed image processing tasks. However the image sensor exposure and data transfer of every frame consume large amount of time and instruction cycles thus greatly reduce the processing rate of our vision system.

In this paper, we apply a superscalar PE to our previous architecture. The new PE structure is capable of simultaneously executing an image transfer instruction and an image processing instruction, thus frame pipeline is achieved. A calculated PE performance improvement is nearly $100 \%$ for some algorithms. A parallel computing language and its compiler and assembler are developed to support the new PE programming and related further designs.

This paper proceeds as follows. In section II, we will describe the architecture of our vision system, the new $\mathrm{PE}$ structure and the parallel computing language. In sec- tion III, the FPGA implementation is presented. And finally we draw conclusion in section IV.

\section{Architecture of the System}

\subsection{System Architecture}

The architecture of the proposed vision system is presented in fig.1. It consists of a pixel-parallel PE array, a row-parallel processor, a RISC core, an on-chip AHB bus, a sensor controller and an I/O module. The sensor interface is responsible for sub sampling the image plane. The PE array is composed of $\mathrm{M} \times \mathrm{M}$ identical PEs, each $\mathrm{PE}$ is a single bit processor connected with its up, down, left, right PE neighborhoods. The row-parallel processors serve as the interface of PE array with the RISC core and carry out middle-level image processing. The RISC core controls the whole system and performs high-level image processing.

In summary, the system architecture integrates three different kinds of processors targets at different levels of image processing. It is specifically designed for high-speed image processing.

\subsection{PE Structure}

Every PE cell is connected to its nearest four neighbors in four directions: up, right, down, left. All PEs receive the same instructions and operate in an SIMD fashion. $\mathrm{PE}$ is built in accumulator architecture [3] that one ope- 
rand is implicit and another operand is explicit. It consists

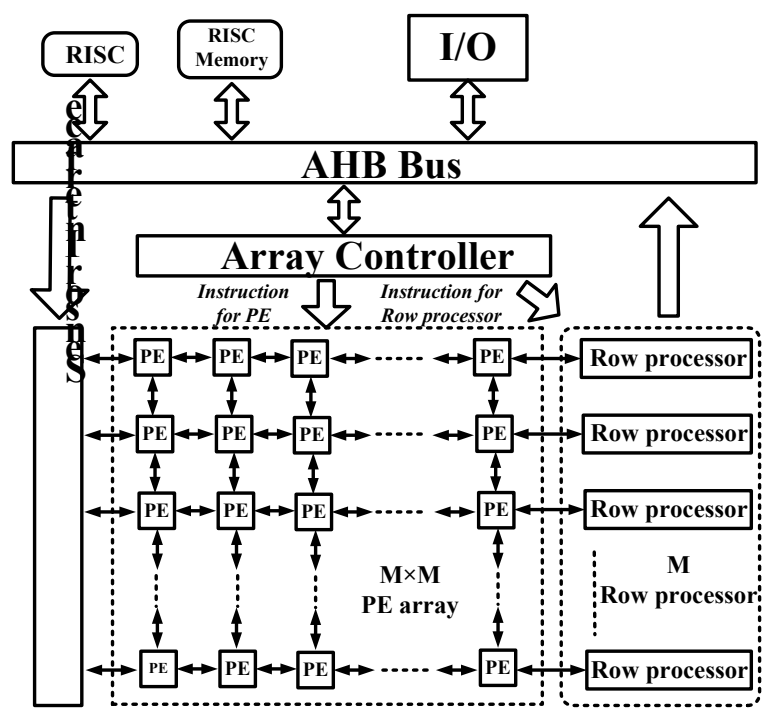

Figure 1. The vision system architecture.

of a 1-bit ALU which can perform basic operation including addition, inversion, and, or, two bank memory, a channel controller and some multiplexers. Both our pervious PE structure and superscalar PE are shown in Figure 2 .
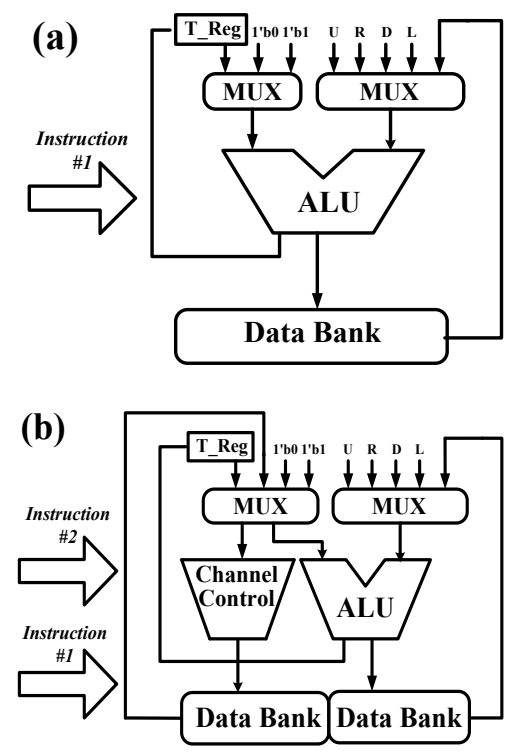

Figure 2. Comparison of PE structure: (a) Our Previous PE structure; (b) superscalar PE structure.

In our previous designs, when captured image data is transferred between PEs the data occupy the data-path, thus makes the PE stall for data processing. To overcome this difficulty, we implemented an individual data-path by adding a channel controller and a data bank. Comparison of the efficiency of superscalar PE with previous PE is shown in Figure 3. Our previous PE is stalled until the $\mathrm{n}_{\text {th }}$ frame is completely captured and transferred into the PE array. The superscalar PE can processing the $n-1_{\text {th }}$ frame while capture and transfer the $\mathrm{n}_{\text {th }}$ frame simultaneously, both the image sensor exposure time and transfer time are concealed.

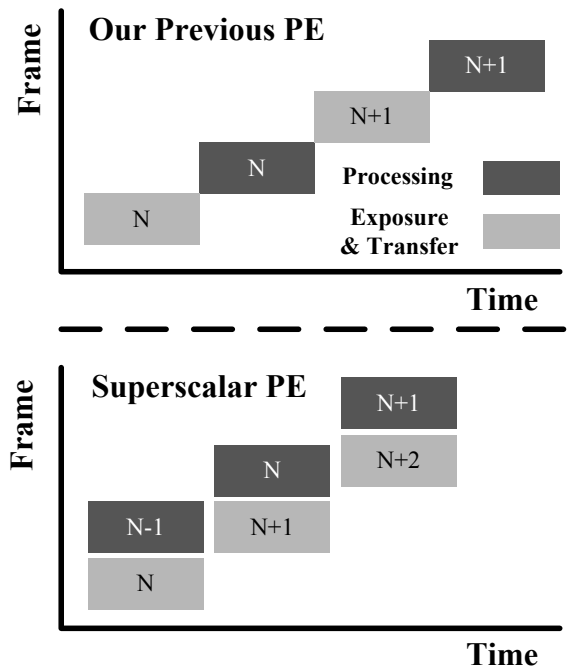

Figure 3. The PE structure

Figure 3. Working efficiency of different PE structure

A simple benchmark for both type of PE structure is shown in Figure 4.

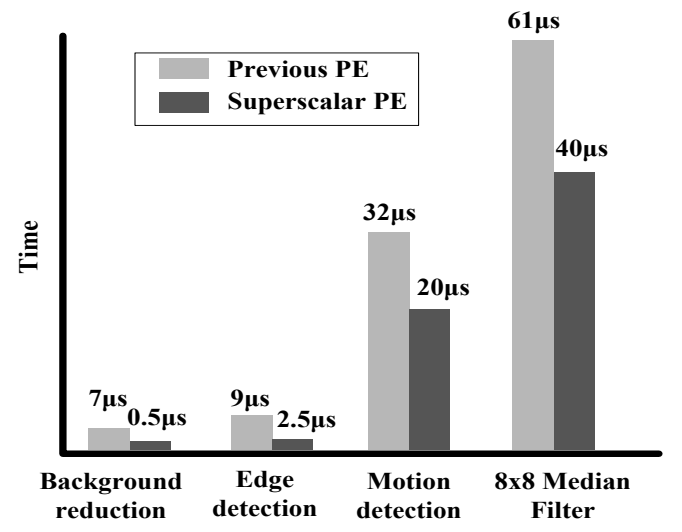

Figure 4. Performance comparison

\subsection{Programming Language}

Both PE and row-processor instruction sets are carefully designed to support low- and middle-level image processing algorithms. Application developing has to be based on those instruction sets. In order to achieve high flexibility and reduce developing time, a parallel compu- 
ting language and its compiler and assembler are developed. The separation of compiler and assembler enables us to alter our instruction encoding format in further designs without greatly changing our compiler. The compile, assemble flow is shown in Figure 5.

After the compiler finishes lexical analysis, parsing, ASM code is generated and passed to the assembler, and then the assembler creates executable file based on our instruction set. The RISC code is compiled by commercial C compiler.

\section{FPGA Implementation}

We utilize a high-speed commercial camera and Altera Cyclone III FPGA to implement our vision system. Due to limited on chip resources we choose $64 \times 64$ as PE array size. The commercial camera can work at $1000 \mathrm{fps}$, we store the image captured by camera into the FPGA SRAM, and then the processor fetches image data from the SRAM through the sensor interface. Note that the data in the SRAM are always available for the processor,

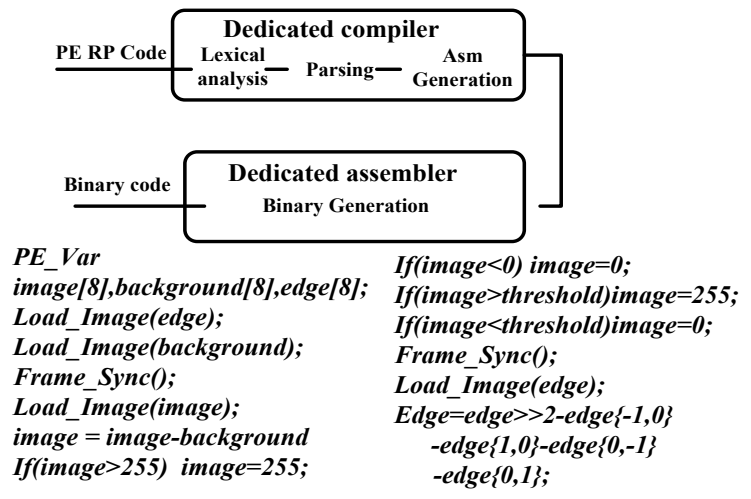

Figure 5. The compile flow and parallel computing language.

as if it is an ideal sensor with infinite frame rate. So the max processing rate can be obtained by measuring the frame rate of the vision system. The clock frequency of the vision system is $100 \mathrm{MHz}$, the performance is about 44GOPS when 8-bit addition is performed, and the throughput of the PE array is $50 \mathrm{~GB} / \mathrm{s}$. A moving detection result is shown in Figure 6, (a) is the background image and (b) is an image with a moving object, the white line box roughly indicates the moving region.
Great performance improvement is achieved for low- and middle-level image processing due to the implementation of superscalar PE. The measured processing rate for moving detection is $2000 \mathrm{fps}$ for $256 \times 256$ resolution image.

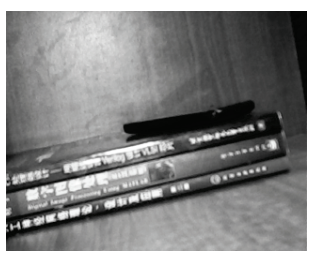

(a)

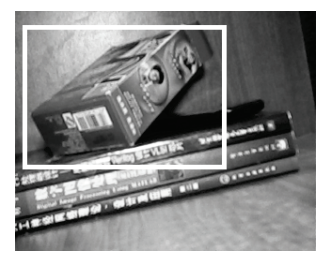

(b)
Figure 6. Result of moving detection

\section{Conclusion}

This paper describes a FPGA prototype of a programmable vision system implementing in Altera Cyclone III FPGA. Its parallel architecture fully covered and optimized for low-, middle-, high-level image processing. With our dedicated parallel computing language the vision system is capable of performing various image processing algorithms. Our final implementation includes a PE array of $64 \times 64$ targeted at low-level image processing, 64 row processors targeted at middle-level image processing and a RISC core for high-level image processing and system control. The clock frequency of the vision system is $100 \mathrm{MHz}$. it can achieve motion detection at a rate of $2000 \mathrm{fps}$ with resolution of $256 \times 256$, and $104 \mathrm{fps}$ face detection task. The results demonstrated that our vision system is suitable for various high-speed real-time required image processing applications.

\section{REFERENCES}

[1] T. Komuro, S. Kagami, and M. Ishikawa, "A Dynamically Reconfigurable SIMD Processor for a Vision Chip," IEEE Journal of Solid-State Circuits, Vol. 39, No. 1, 2004. doi: 10.1109/JSSC.2003.820876

[2] W .C. Zhang, Q. Y. Fu, and N. J. Wu, “ A Programmable Vision Chip Based on Multiple Levels of Parallel Processors," IEEE Journal of Solid-State Circuits, Vol. 46, No. 9, 2011. doi: 10.1109/JSSC.2011.2158024

[3] J. Hennessy, D.A. Patterson, "Computer Architecture: A Quantitative Approach," 5th Edition, Morgan Kaufmann, San Francisco, CA, 2011. 